\title{
MULTIPLE LEVEL FINITE ELEMENT \\ ANALYSIS FOR TIDAL CURRENT FLOW \\ WITH NON-REFLECTIVE OPEN \\ BOUNDARY CONDITION
}

\author{
Toshio KODAMA* and Mutsuto KAWAHARA**
}

\begin{abstract}
A finite element method for the multiple level analysis of tidal current flow considering an open boundary condition is presented in this paper. The incident wave condition which does not cause spurious reflective waves on the open boundary has been proposed and it has been applied to the boundary condition in the multiple level tidal current analysis. To determine the reflective wave component, the subdomain located adjacent to the open boundary is introduced. The reflective wave component on the open boundary can be extracted by computing on the subdomain. The numerical results are compared with the analytical solution and the observed data. Both results show well in agreement.

Keywords : finite element method, tidal current analysis, multiple level model, open boundary condition
\end{abstract}

\section{INTRODUCTION}

In the analysis of tidal current flow based on the finite difference method or finite element method, a flow field is surrounded by both land and artificial boundaries, the latter of which is often called as an "open boundary" and located at the offshore side. It is conventional that the perfect or partial reflective condition is imposed on the land boundary and the periodical water surface elevation is specified on the open boundary in case of closed type bay in the calculation of transient flow. Under such boundary condition and the appropriate initial condition in which the velocity and water elevation are usually assumed to be zero, the linear or nonlinear shalllow water equation is numerically computed. Numerous studies based on the above situations have been performed ${ }^{1)-3)}$.

However, in the strict sense, the boundary condition on which only the periodical water elevation is imposed, is incorrect for the analysis with 'cold start' condition, because the spurious reflection is occured by the initial transient flow on the open boundary, and the reflective wave introduces the mode of free oscillation. The outgoing waves should be freely transmitted through the open boundary without such spurious reflections. In almost all of computation employed in the practical use, this condition is not usually satisfied. Thus, special numerical treatment which should avoid these phenomena is required to the open boundary condition.

\footnotetext{
* Member of JSCE, M. Eng., Assistant, Department of Civil Engineering Chuo University (Kasuga 1-13-27, Bunkyo-ku, Tokyo 112)

** Member of JSCE, Dr. Eng., Professor, Department of Civil Engineering Chuo University
}

In this paper, the issue of open boundary treatment will be made clearly through the simulation of wave propagation problem in one dimensional bay model. Then the literatures dealing with the open boundary condition which overcome the problems are reviewed and the new treatment method on the basis of the finite element method is proposed for the multiple tidal current analysis. The method is verified by the numerical simulation using a one dimensional channel. It is shown that spurious reflective wave has completely been eliminated by the present method. Finally, the tidal current analysis of Tokyo Bay is carried out to show the applicability to the practical analysis. The comparison between the computed results and observed data will be shown.

\section{MULTIPLE LEVEL MODEL}

Multiple level analyses of the current flow have been performed by the finite difference method $^{4) \sim 10)}$. The research by Wang ${ }^{11)}$ is probably the first to present multiple level flow analysis by the finite element method. However, the vertical velocity has been ignored in the above three dimensional analysis. Kobayashi et al. ${ }^{12)}$ extended the method to include the vertical velocity component. The analysis by Kobayashi et al. ${ }^{12}$ is limited to steady state computation. Almost all the finite element analyses developed recently employ time marching schemes for the numerical integration in time. Kawahara et al. ${ }^{2)}$ dealt with the finite element method with the time marching scheme for the multiple level analysis of a constant density current flow in the case of tidal motion.

In this study the multiple model proposed by Kawahara et al. ${ }^{2)}$ is employed as the mathematical model. The basic equations of the multiple level 
flow are expressed as the following conservation equations of momentum and continuity :

$$
\begin{aligned}
& \frac{\partial u}{\partial t}+\frac{\partial}{\partial x}(u u)+\frac{\partial}{\partial y}(u v)+\frac{\partial}{\partial z}(u w) \\
& =f v-\frac{1 \partial p}{\rho \partial x}+\frac{1}{\rho}\left(\frac{\partial \tau_{x x}}{\partial x}+\frac{\partial \tau_{x y}}{\partial y}+\frac{\partial \tau_{x z}}{\partial z}\right) \\
& \frac{\partial u}{\partial t}+\frac{\partial}{\partial x}(u v)+\frac{\partial}{\partial y}(v v)+\frac{\partial}{\partial z}(v w) \\
& =-f v-\frac{1 \partial p}{\rho \partial y}+\frac{1}{\rho}\left(\frac{\partial \tau_{y x}}{\partial x}+\frac{\partial \tau_{y y}}{\partial y}+\frac{\partial \tau_{y z}}{\partial z}\right) \\
& \frac{\partial p}{\partial z}+\rho g=0 \\
& \frac{\partial u}{\partial x}+\frac{\partial v}{\partial y}+\frac{\partial w}{\partial z}=0
\end{aligned}
$$

Where $x, y, z$ represent Cartesian coordinates positive eastwards, northwards and upwards respectively, $t$ is time, $u, v, w$ denote velocity components for $x, y, z$ and $p, f$ are pressure and Coriolis parameter respectively. The approximation of hydrostatic pressure distribution is employed for the momentum equation of vertical direction. The density of water, $\rho$ is treated as a constant in the horizontal direction postulating that water is incompressible. Turbulent shear stress is expressed by $\tau_{x x}, \tau_{x y}, \tau_{x z}, \tau_{y y}, \tau_{y z}$ and is assumed to be a function of velocity gradient.

The level system is introduced in the vertical water depth direction as shown in Fig.1. A representative level in the level system is referred to as $L^{(k)}$, where $L^{(k)}$ denotes the negative value of $z$ measured from the mean water elevation. The surface level is expressed by $L^{(1)}$ and the bottom level is $L^{(b)}$. Henceforth, the indicial notation and the summation convention with repeated indices will be employed in order to simplify the expression. The subscript ()$_{, i}$ indicates the partial differentiation with respect to the $i$ coordinate. The symbols $\delta_{i j}$ and $\epsilon_{i j}$ are Kronecker's delta and Edington's epsilon function respectively.

Integrating the horizontal velocity over the $(k)$ th water volume held between $L^{(k-1)}$ and $L^{(k)}$, the vertically averaged horizontal velocity is defined as follows :

$$
u_{i}^{(k)}=\frac{1}{h^{(k)}} \int_{L^{(k-1)}}^{L^{(k)}} u_{i} d z
$$

The $(k)$ th water volume thickness is expressed as follows :

$$
h^{(k)}=L^{(k)}-L^{(k-1)}
$$

The vertically averaged equations of momentum and continuity are defined from Eqs. (1)-(4) using (5). The equations of momentum can be expressed

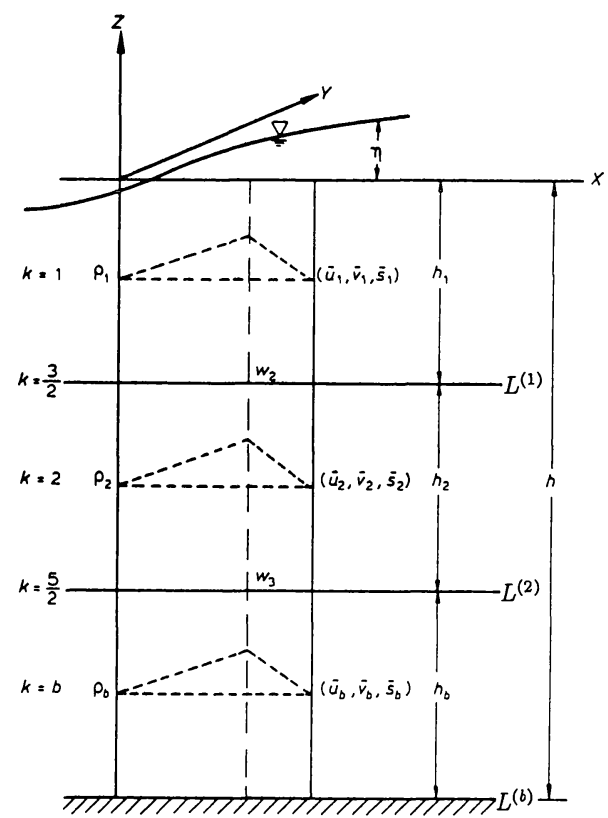

Fig.1 Level system

as follows :

$$
\begin{aligned}
& \frac{\partial u_{i}{ }^{(k)}}{\partial t}+u_{j}{ }^{(k)} u_{i, j}{ }^{(k)}+\frac{1}{h^{(k)}}\left\{\left(u_{i} w\right)^{(k-1 / 2)}\right. \\
& \left.-\left(u_{i} w\right)^{(k+1 / 2)}\right\}+\frac{1}{\rho^{(k)}} p_{, i}{ }^{(k)}-f \epsilon_{i j} u_{j}{ }^{(k)} \\
& =\frac{1}{\left(\rho^{(k)} h^{(k)}\right)}\left\{h^{(k)}\left(\tau_{i j, j}{ }^{(k)}\right)+\left(\tau_{i}^{(k) u}-\tau_{i}^{(k)}\right)\right\}
\end{aligned}
$$

Where $\rho^{(k)}$ is the water density in the $(k)$ th water volume, $p^{(k)}$ is the pressure computed by :

$$
p^{(k)}=\rho^{(1)} g \eta-\sum_{m=1}^{k-1} \rho^{(m)} g h^{(m)}-\rho^{(k)} g\left(z-\sum_{m=1}^{k-1} h^{(m)}\right)
$$

Where $\eta$ is the water elevation measured from the mean water elevation, the term $\left(u_{i} w\right)^{(k-1 / 2)}$ is :

$$
\left(u_{i} w\right)^{(k-1 / 2)}=w^{(k)} \cdot \frac{1}{2}\left(u_{i}{ }^{(k)}+u_{i}{ }^{(k-1)}\right)
$$

Where $w^{(k)}$ is the vertical velocity component computed in the $(k)$ th water volume.

The equation of continuity can be written for the surface level as :

$$
\frac{\partial \eta}{\partial t}+\sum_{l=1}^{b}\left(h^{(l)} u_{i}^{(l)}\right)_{, i}=0
$$

The continuity equation for the middle layer is :

$$
w^{(k)}=-\sum_{l=k}^{b}\left(h^{(l)} u_{i}^{(l)}\right)_{, i}
$$

The turbulent shear stress is assumed as :

$$
\tau_{i j}{ }^{(k)}=A_{l}{ }^{(k)}\left(u_{i, j}{ }^{(k)}+u_{j, i}{ }^{(k)}\right)
$$


cient. The interface shear stress is modelled using the quadratic friction law. For the surface level, the wind friction is introduced in the from :

$$
\tau_{i}^{(1) u}=c^{*} \rho_{a} W W_{i}
$$

where $c^{*}, \rho_{a}, W, W_{i}$ are wind drag coefficient, air density, magnitude of wind velocity and wind velocity respectively. For the intermediate level, the following friction are used :

$$
\begin{aligned}
& \tau_{i}^{(k) u}=\frac{f_{m}}{2 h^{(k)}}\left(\rho^{(k-1)}+\rho^{(k)}\right)\left(u_{i}{ }^{(k-1)}-u_{i}{ }^{(k)}\right) \Delta V^{(k)}, \\
& \tau_{i}^{(k) l}=\frac{f_{m}}{2 h^{(k)}}\left(\rho^{(k)}+\rho^{(k+1)}\right)\left(u_{i}^{(k)}-u_{i}{ }^{(k+1)}\right) \Delta V^{(k)}
\end{aligned}
$$

where $f_{m}$ is a friction coefficient and :

$$
\Delta V^{(k)}=\left\{\left(u_{i}{ }^{(k-1)}-u_{i}{ }^{(k)}\right)\left(u_{i}{ }^{(k-1)}-u_{i}{ }^{(k)}\right)\right\}^{1 / 2} .
$$

For the bottom level, the bottom friction :

$$
\tau_{i}^{(b) l}=\frac{f_{b}}{h^{(b)}} \rho^{(b)} u_{i}{ }^{(b)}\left(u_{k}{ }^{(b)} u_{k}{ }^{(b)}\right)^{1 / 2}
$$

is used where $f_{b}$ is the friction coefficient of bottom.

The following two types of boundary conditions are considered. On the land boundary $\Gamma_{L}$, at the $(k)$ th level, the velocity is assumed as :

$$
u_{n}{ }^{(k)}=0 \text { on } \Gamma_{L} \text {. }
$$

where $n$ denotes a normal direction to the bonudary. On the open bonudary $\Gamma_{0}$, the following boundary condition is imposed.

$$
\bar{u}_{n}{ }^{(k)}=\frac{g}{C}\left(\bar{\eta}^{I} n^{+}+\bar{\eta}^{R} n^{-}\right) \text {on } \Gamma_{0}
$$

were $C=\sqrt{g h}$ denotes the phase speed, $I$ and $R$ refer to the incident and reflective wave component respectively, the overbar indicates that values on the open boundary and symbols $n^{+}$and $n^{-}$mean unit normal vector in a positive and negative direction to the boundary. Arbitrary wave direction of incident wave can be considered straightforward by shifting the vector $n^{+}$included in Eq. (18). In a strict sense, the wave direction of the reflective wave is essentially unknown and the direction will be affected by the configuration of bay or location of the bounday. However, in case that wave length is much larger than the scale of bay, it is natural way that the direction of the reflective wave is assumed as the normal to the boundary. Thus, the wave direction of the reflective wave is taken as the normoal to the open boundary in this study.

The incident wave condition is given as the known function,

$$
\bar{\eta}^{I}=\sum_{m=1}^{N c} a_{m} \sin \left(k_{n}{ }^{m} n-\omega_{m} t-\kappa_{m}\right)
$$

where $a_{m}, k_{n}{ }^{m}, \omega_{m}$ and $\kappa_{m}$ mean amplitude, wave
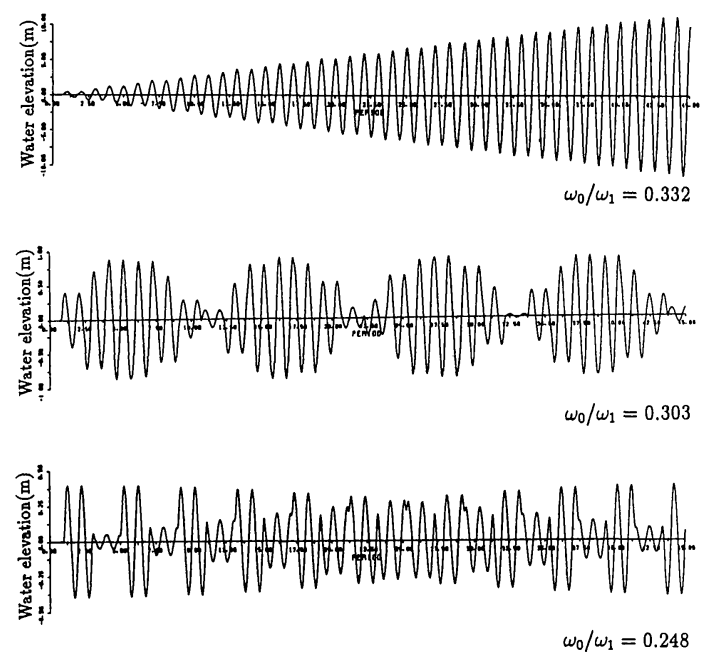

Fig.2 Response of the water elevation

number, angular frequency and phase delay respectively and $N_{c}$ is a number of constituents. The tidal wave is assumed as the sum of one dimensional functions. Reflective wave, $\bar{\eta}^{R}$ is the unknown. The procedure in which the reflective wave component is determined and the open boundary condition to be imposed are precisely explained in the following section.

\section{ISSUE OF OPEN BOUNDARY TREATMENT}

Qian et al. ${ }^{13)}$ have shown the following facts by using the analytical solution of one dimensional linear shollow water equation. The conventional boundary condition has used the periodical water elevation as a condition, which is incorrect for the analysis with 'cold start' condition. Because the spurious reflection is occured by the initial transient flow on the open boundary, and it introducse the mode of free oscillation. Fig.2 shows the response modes of water elevation at interior of the bay by the conventional method. In this figure, vertical and horizontal axes mean water elevation and period (time) respectively. The angular frequency of incident wave and natural frequency of the bay are expresed by $\omega_{0}$ and $\omega_{1}$. It is shown that the phenomena such as divergence, beat, instability are observed according to the ratio of the frequency of incident wave and natural frequency of the bay. The reason, these phenomena have been occured is that the free oscillation mode is added to the periodical component. In the tidal analysis, the effect of this free oscillation is relatively small because of the reason ; (1) The friction terms such as the bottom friction and artificial viscosity, etc. may reduce the 
oscillation ; (2) The period of tidal wave is much longer than that of eigen period of the bay. However, it is impossible to eliminate this free oscillation mode under the conventional boundary condition.

Generally the boundary condition mentioned above is discussed in the initial boundary value problems for the system of hyperbolic equation. Theoretical and numerical methods have been presented in the short wave problem, e. g., Smith $^{14)}$, Oliger and Sundstorm ${ }^{15)}$, Engquist and $\mathrm{Majda}^{16)}$. For the long wave problem, the literatures which show the numerical treatment of the open boundary condition and the practical application such as tidal current is limited. Bode and Sobey ${ }^{17)}$ have investigated the effects of the free oscillation by the method of characteristics and presented a method of imposing radiation boundary condition on the open boundary, and applied this to the wave propagation problem in one dimensional channel by the characteristics algorithm. Blumberg and Kanth ${ }^{18)}$ have also applied the radiation condition to the open boundary in the finite difference method and used this to practical problems. The method of characteristics with the radiation condition employed in those methods may be effective, but it is relatively difficult to apply this to the finite element analysis because this method can be suitable for the case of regular staggered grid. Contrary to this, the method may create more complicities in the computation for arbitrary configuration.

Therefore, the present paper mainly aims at the long wave analysis by the finite element method and presents a new treatment of the open boundary condition for the periodical flow analysis such as tidal current. Then the present method will be applied to the tidal current analysis using multiple phase model.

\section{TREATMENT OF OPEN BOUNDARY CONDITION}

In this section, the computational procedure to treat the open boundary condition is shown. The conceptual figure of finite element discretization is shown in Fig.3 (a). Along the open boundary on which the coordinate $s$ is located, and $n$ is normal to the boundary. The line I-I $\left(n=n_{0}\right)$ corresponds to the open boundary. The line II - I $\left(n=n_{0}\right.$ $+\Delta n)$ is placed on a single layer of the finite elements inside the domain. Fig.3 (b) shows the subdomain which will be used to compute the reflective wave component at the open boundary $\mathrm{I}-\mathrm{I}$.

It is assumed that the incident wave condition, $i$. e. water elevation can be expressed as a linear

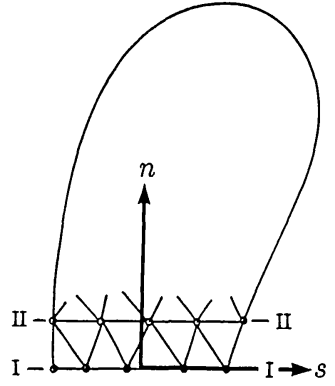

(a) Whole-domain

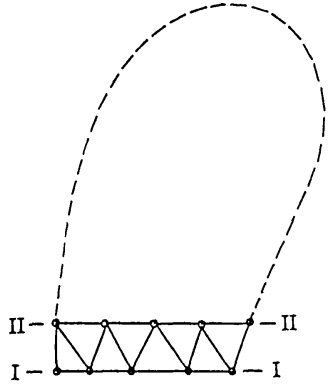

(b) Sub-domain
Fig.3 Conceptual figures of the finite element idealization

combination of the incident and reflective wave components as follows :

$$
\bar{\eta}\left(n_{0}, s, t\right)=\bar{\eta}^{I}\left(n_{0}, s, t\right)+\bar{\eta}^{R}\left(n_{0}, s, t\right)
$$

and the boundary condition have to be imposed as follows :

$$
\bar{u}_{n}^{(k)}\left(n_{0}, s, t\right)=\frac{g}{C}\left\{\bar{\eta}^{I}\left(n_{0}, s, t\right) n^{+}+\bar{\eta}^{R}\left(n_{0}, s, t\right) n^{-}\right\} .
$$

The incident wave condition is assumed to be known function expressed as :

$$
\bar{\eta}^{I}\left(n_{0}, s, t\right)=a_{I} \sin \left(k_{n} n_{0}-\omega t-\kappa\right)
$$

In case of treating the short wave problem, an angle of incident wave is much effective to the solutions in the region to be analyzed. However, the tidal current which should be computed here is long wave, thus, it is not hindered to consider that the incident wave is coming from the normal direction to the open boundary. Obviously, the tide is periodical phenomenon and the incident wave can be assumed as the sum of the harmonic functions like a sinusoidal wave.

It is required to determine the reflective wave component which is essentilly unknown. The logic of this treatment is explained as follows.

At first, the initial values of variables at all nodal points are set to be zero.

Second, in the whole domain, the variables at all nodal points are calculated using the incident wave condition given by Eq. (19) with the boundary conditions. In that time, computed values includes both incident and reflective component. In parallel, in the subdomain, the incident wave component at one mesh size inside from the open boundary, i. e. line II-II can be computed under the same incident wave condition. Here, on the outlet boundary, the progressive wave condition is imposed. Then, the reflective component at line II - II can be calculated taking the difference between the component obtained by the whole domain and the incident wave component obtained by the 
subdomain.

Third, since the reflective wave component could be obtained on line II-II, the reflective component which would propagate toward to the open boundary (line I-I) can be computed using subdomain again. The progressive wave condition is also applied to the outlet boundary.

Finally, the open boundary condition which should be imposed at the next time iteration, can be calculated taking the sum of the incident and reflective components which have been obtained by the above procedure. Accordingly the subdomain is introduced as dummy to obtain the reflective wave component. The procedure of the computation for the open boundary condition is shown below :

[Step 0] Set $u_{\imath}{ }^{(k)}\left(n, s, t_{0}\right)=0.0$ and $\eta\left(n, s, t_{0}\right)=$ 0.0. for all nodal points.

[Step 1] Compute $u_{i}{ }^{(k)}(n, s, t+\Delta t)$ and $\eta(n, s, t+$ $\Delta t)$ in the whole domain with the incident wave conditions, $\bar{\eta}^{I}\left(n_{0}, s, t\right)$ and boundary condition :

$$
\bar{u}_{n}^{I(k)}\left(n_{0}, s, t\right)=\frac{g}{C} \bar{\eta}^{I}\left(n_{0}, s, t\right) n^{+} \text {. }
$$

[Step 2] Compute $u_{n}{ }^{I(k)}\left(n_{0}+\Delta n, s, t+\Delta t\right)$ and $\eta^{I}$ $\left(n_{0}+\Delta n, s, t+\Delta t\right)$ in the subdomain with the incident wave conditions, $\bar{\eta}^{I}\left(n_{0}, s, t\right)$ and boundary conditions :

$$
\bar{u}_{n}^{I(k)}\left(n_{0}, s, t\right)=\frac{g}{C} \bar{\eta}^{I}\left(n_{0}, s, t\right) n^{+}
$$

and

$$
\begin{aligned}
\bar{u}_{n}{ }^{I(k)} & \left(n_{0}+\Delta n, s, t+\Delta t\right) \\
& =\frac{g}{C} \eta^{I}\left(n_{0}+\Delta n, s, t+\Delta t\right) n^{+} .
\end{aligned}
$$

[Step 3] Compute components of the reflective waves :

$$
\begin{aligned}
& u_{n}{ }^{R(k)}\left(n_{0}+\Delta n, s, t+\Delta t\right)=u_{n}{ }^{(k)}\left(n_{0}+\Delta n, s, t+\Delta t\right) \\
& \quad-u_{n}{ }^{I(k)}\left(n_{0}+\Delta n, s, t+\Delta t\right), \\
& \eta^{R}\left(n_{0}+\Delta n, s, t+\Delta t\right)=\eta\left(n_{0}+\Delta n, s, t+\Delta t\right) \\
& \quad-\eta^{I}\left(n_{0}+\Delta n, s, t+\Delta t\right) .
\end{aligned}
$$

[Step 4]. Set $t=t+\Delta t$

[Step 5] Compute $\bar{u}_{n}{ }^{R(k)}\left(n_{0}, s, t+\Delta t\right)$ and $\bar{\eta}^{R}$ $\left(n_{0}, s, t+\Delta t\right)$ in the subdomain with the incident wave condition, $\eta^{R}\left(n_{0}+\Delta n, s, t\right)$ and boundary conditions :

$$
u_{n}^{R(k)}\left(n_{0}+\Delta n, s, t\right)=\frac{g}{C} \eta^{R}\left(n_{0}+\Delta n, s, t\right) n^{-}
$$

and

$$
\bar{u}_{n}^{R(k)}\left(n_{0}, s, t+\Delta t\right)=\frac{g}{C} \bar{\eta}^{R}\left(n_{0}, s, t+\Delta t\right) n^{-} .
$$

[Step 6] Compute the open boundary condition for the whole domain :

$$
\bar{u}_{n}^{(k)}\left(n_{0}, s, t+\Delta t\right)=\frac{g}{C} \bar{\eta}^{I}\left(n_{0}, s, t+\Delta t\right) n^{+}
$$

$$
+\frac{g}{C} \bar{\eta}^{R}\left(n_{0}, s, t+\Delta t\right) n^{-}
$$

and incident wave condition :

$$
\bar{\eta}\left(n_{0}, s, t+\Delta t\right)=\bar{\eta}^{I}\left(n_{0}, s, t+\Delta t\right)+\bar{\eta}^{R}\left(n_{0}, s, t+\Delta t\right) .
$$

[Step 7] Repeat computaions starting from Step 1.

Applying this procedure spurious reflection wave is completely eliminated on the open boundary. The treatment of the present method is carried out in local, i.e.in the subdomain which has nodal points on the open boundary, it is applicable to the implicit scheme in time, but, it will take much time to compute in the practical computation.

\section{FINITE ELEMENT METHOD}

The conventional Galerkin methods is employed for the finite element formulation. Horizontal velocity components and water elevation for the $(k)$ th level are interpolated in each finite element :

$$
u_{i}{ }^{(k)}=\Phi_{\alpha} u_{\alpha i}{ }^{(k)}, \eta=\Phi_{\alpha} \eta_{\alpha}
$$

where $\Phi_{\alpha}$ denotes the interpolation function for velocity or water elevation, and $u_{\alpha i}{ }^{(k)}, \eta_{\alpha}$ mean velocity and water elevation at the $\alpha$ th node of each finite element respectively. The interpolation function, a standard linear function based on the three node triangular element is used in this paper. The Galerkin procedure leads to the following finite element equation for the $(k)$ th level, i. e. for equation of momentum :

$$
\begin{aligned}
& M_{\alpha \beta} \dot{u}_{\beta i}^{(k)}+K_{\alpha \beta \gamma j} u_{\beta}^{(k)} u_{r_{t}}^{(k)}+f \epsilon_{i j} M_{\alpha \beta} u_{\beta j}^{(k)} \\
& +N_{\alpha_{i} \beta} p_{\beta}^{(k)}+\frac{A_{l}}{\rho^{(k)}} \cdot S_{\alpha i \beta j} u_{\beta j}^{(k)}-\left(T_{\alpha i}^{(k) u}-T_{\alpha i}^{(k) l}\right) \\
& -\Pi_{\alpha i}^{(k)}=0 \ldots \ldots \ldots \ldots \ldots \ldots \ldots \ldots \ldots \ldots \ldots \ldots \ldots \ldots \ldots \ldots \ldots \ldots \ldots \ldots \ldots \ldots \ldots \ldots \ldots
\end{aligned}
$$

for the equation of continuity for surface level :

$$
M_{\alpha \beta} \dot{\eta}_{\beta}+\sum_{q=1}^{b}\left(h^{(q)} N_{\alpha_{i} \beta} u_{\beta_{t}}{ }^{(q)}\right)=0
$$

and for the equation of continuity for intermediate level :

$$
M_{\alpha \beta} w_{\beta}{ }^{(k)}=-\sum_{l=k}^{b}\left(h^{l} N_{a_{t} \beta} u_{\beta_{t}}{ }^{(l)}\right)=0
$$

where :

$$
\begin{aligned}
& M_{\alpha \beta}=\int_{V}\left(\Phi_{\alpha} \Phi_{\beta}\right) d V \\
& K_{\alpha \beta \gamma j}=\int_{V}\left(\Phi_{\alpha} \Phi_{\beta} \Phi_{r, j}\right) d V \\
& N_{\alpha \beta i}=\int_{V}\left(\Phi_{\alpha} \Phi_{\beta, i}\right) d V \\
& S_{\alpha_{t \beta} \beta}=\int_{V}\left(\Phi_{\alpha, i} \Phi_{\beta, j}\right) d V+\int_{V}\left(\Phi_{\alpha, k} \Phi_{\beta, k}\right) \delta_{i j} d V \\
& T_{\alpha i}^{\left({ }^{(k) u}\right.}=\int_{V}\left(\Phi_{a} \tau_{i}^{\left({ }^{(k) u}\right)}\right) d V \\
& T_{\alpha i}{ }^{\left({ }^{\prime}\right)}=\int_{V}\left(\Phi_{a} \tau_{i}^{\left({ }^{(k)}\right)}\right) d V
\end{aligned}
$$




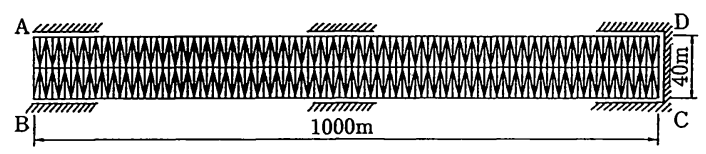

Fig.4 One dimensional channel model

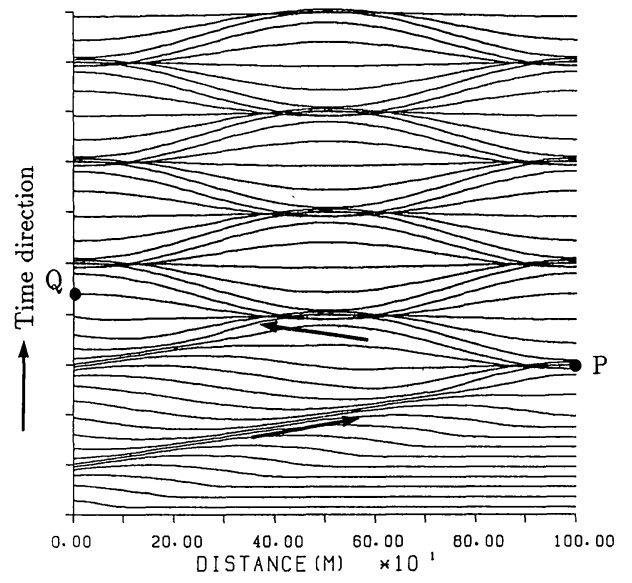

Fig.5 Time variation of water elevation by the present method

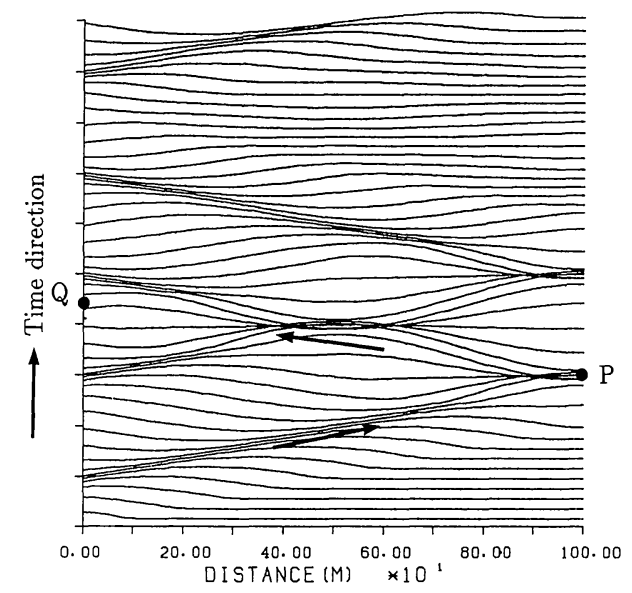

Fig.6 Time variation of water elevation by the conventional method

$$
\Pi_{\alpha i}{ }^{(k)}=\int_{V}\left(\Phi_{\alpha}\left\{\left(u_{i} w\right)^{(k-1 / 2)}-\left(u_{i} w\right)^{(k+1 / 2)}\right\}\right)
$$

To solve the above finite element equations, a numerical integration scheme in time have to be introduced. The integration scheme employed in this paper is termed as the two step explicit scheme $^{19)}$. The open boundary condition described in Section 4 is used for every time step to solve the above element equations.

\section{NUMERICAL EXAMPLES AND DISCUSSIONS}

Two numerical examples are shown to verify the applicability of the present method.
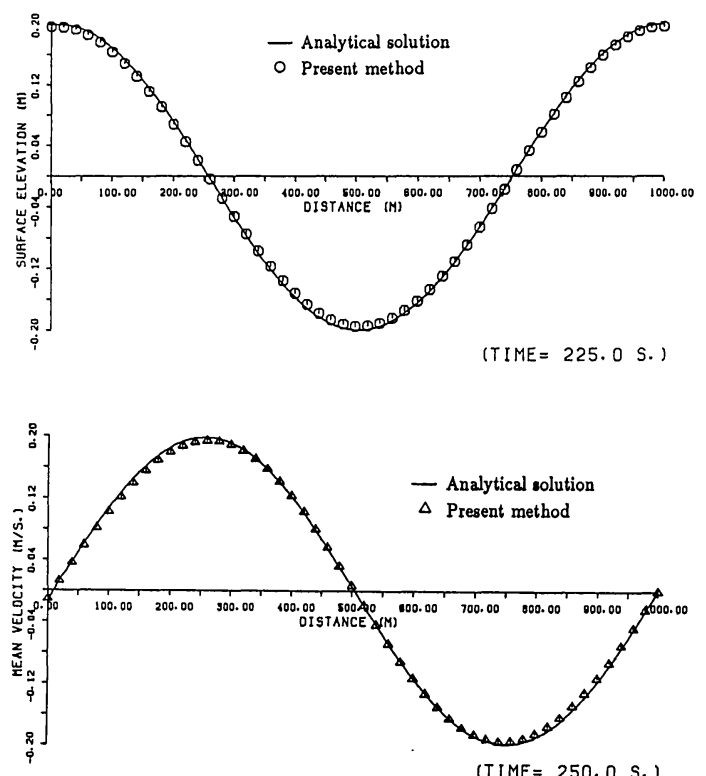

Fig.7 Water elevation and velocity

\section{(1) Standing wave in a one dimensional channel}

The analyses of a standing wave are carried out for the linear equations. The finite element idealization of the one dimensional channel is shown in Fig.4. The total number of nodal points and finite elements of each level is 303 and 400 respectively. On the boundaries A-D and B$\mathrm{C}$, the normal velocities are assumed to be zero. The perfect reflective condition is imposed on the boundary C-D. The boundary A-B is the open boundary on which the incident wave condition formulated in the previous section is imposed. A water depth of the channel is assumed to be $10 \mathrm{~m}$. It is considered that the model consists of five levels having the thickness of $2.0 \mathrm{~m}$. For the incident wave condition, the following sinusoidal functions assumed,

$$
\bar{\eta}^{I}=\alpha_{I} \sin \frac{2 \pi}{T} t
$$

where an amplitude $\alpha_{I}=0.1 \mathrm{~m}$, a period $T=100 \mathrm{~s}$ are employed. The computaion was carried out with the time increment $\Delta t=0.5 \mathrm{~s}$. In this verification, all of the nonlinear terms including Corioli's force are neglected and the density of water is assumed to be 1.0 for all levels because of comparing with analytical solution.

The computed results of water elevation and velocity are shown in Fig.5 7. In Fig.5 the water elevation for subsequent time instants is shown to illustrate the generation of standing waves as superposition of the progressive and reflective waves. The progressive wave generated at the open boundary and propagates toward to the right side wall (Point P) according to the arrow in the figure. This wave reflects at point $P$ and next, propagates toward to the open boundary (Point $Q$ ) superposing with 


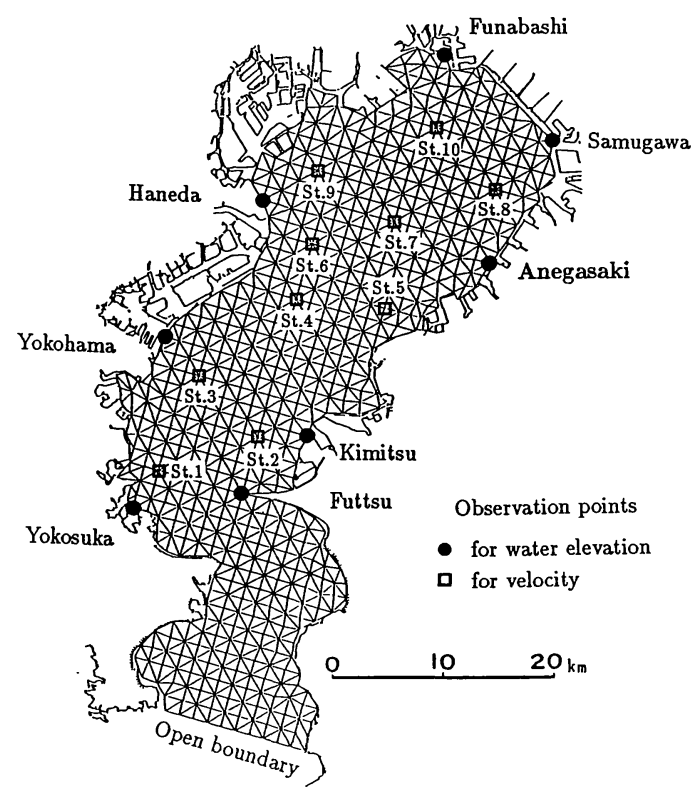

Fig.8 Finite element idealization of Tokyo Bay

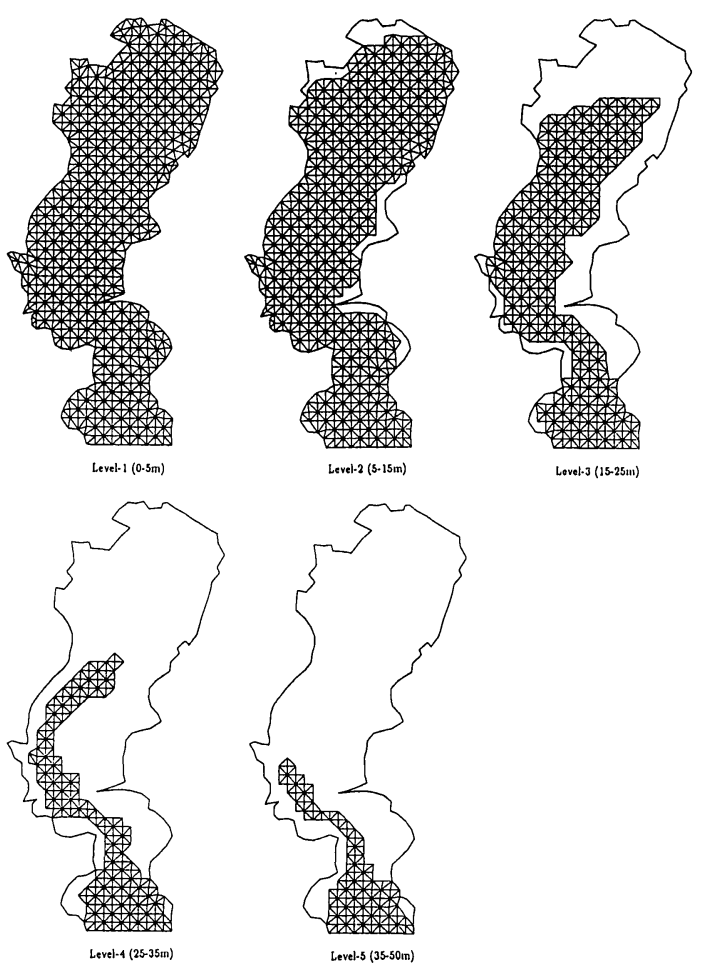

Fig.9 Configuration of water depth

incoming incident wave. The reflect wave reaches at point $\mathrm{Q}$, then, as the reflective wave can pass through the open boundary, after that the filed can be transformed to the standing wave field. In Fig.6 the results for the conventional method, in case that the wave is computed imposing the known function on the open boundary, is
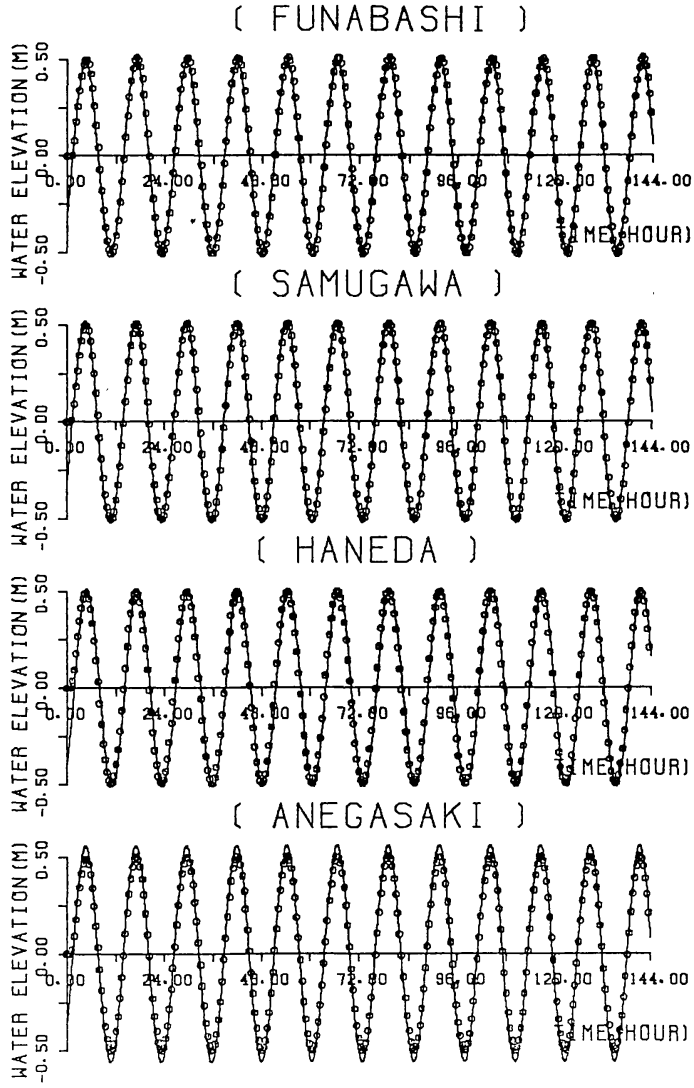

(०) : Present method, (-) : Observed data

Fig.10 Time variation of tide level of $M_{2}$ constituent at the observation points

shown. Until the reflective wave reaches to point $Q$, the computed results are the same as obtained by the present method, whereas the stable field can not be obtained after the wave arrived to the open boundary. It is apparent that the conventional method which imposes only the periodical water elevation on the open boundary causes the spurious reflection and can not simulate the standing waves. The water elevation and velocity for the standing waves obtained are given in Fig.7. The computed results are compared with the analytical solution. The analytical solutions of the water eleveation and velocity were taken as follows :

$$
\begin{aligned}
& \eta(x, t)=2 a_{I} \cos k x \sin \omega t, \cdots \ldots \ldots \ldots \ldots \\
& u(x, t)-\sqrt{\frac{g}{h}} 2 a_{I} \sin k x \sin \left(\frac{\pi}{2}+\omega t\right) .
\end{aligned}
$$

wher $k$ means wave number. The analytical solution were derived from the assumption that the density of each level are identical. The circular and triangular marks in Fig.7 show the computed water elevation and velocity obtained by the preseent method. The computed results by the present method are completely in agreement with the analytical solutions. 

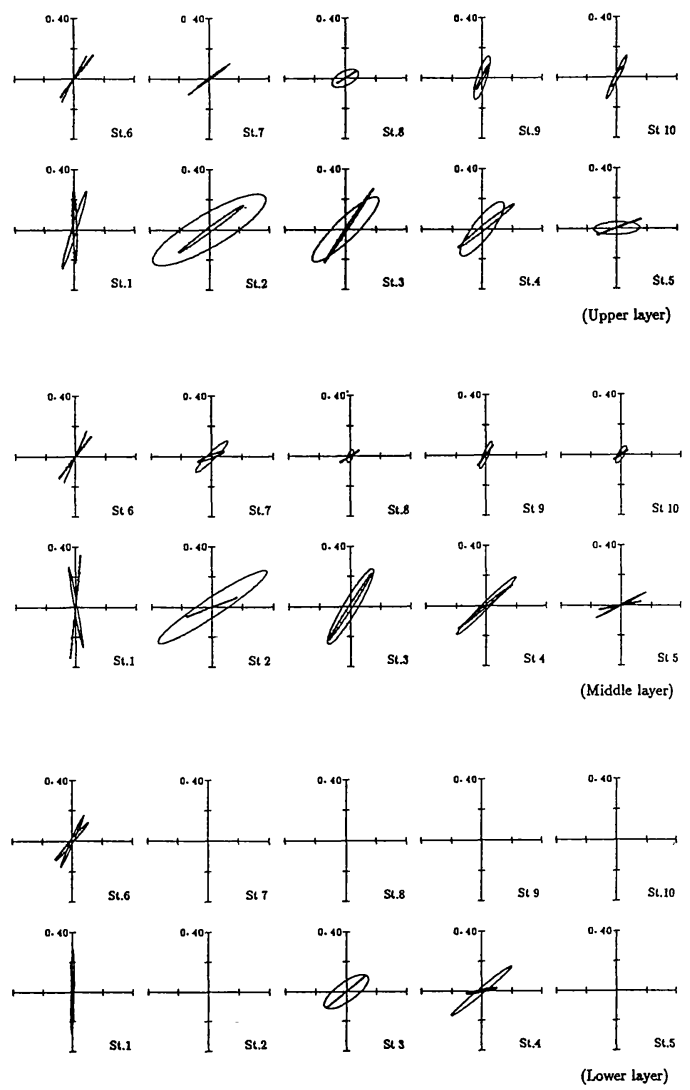

Observed data
Present method, unit : knot

Fig.11 Comparison of tidal ellipses

( 2 ) The tidal current analysis of Tokyo Bay

The simulation of the tidal current in Tokyo Bay is performed to verify the applicability of the present method in a practical problem. The finite element idealization is shown in Fig.8. The flow field is divided into five phase for vertical direction. Total number of nodes and elements of surface level is 685 and 1216, respectively. The tide gauge records and velocity have been measured at points shown in Fig.8. The configuration of water depth of Tokyo Bay is modeled as shown in Fig.9 with reference to the Maritime Chart of Tokyo Datum $^{20)}$. Where the friction coefficient of the interface, $f_{m}$ of 0.001 , the friction coefficient of the bottom. $f_{b}$ of 0.0023 , the coefficient of the eddy viscosity, $A_{l}$ of 10.0 $\mathrm{m}^{2} / \mathrm{s}$. are assumed and the effect of the friction between water surface and wind flow is neglected in this computation. Referring to the water density, from surface, $\rho=1.01,1.015,1.020,1.025,1.030 \times 10^{3} \mathrm{~kg} / \mathrm{m}^{3}$ are assumed. These coefficients are determined referring to the literature 3). Two computations are carried out. The $M_{2}$ constituent is imposed on the open boundary in the first case. The main four constituents of the tide, i. e. $M_{2}$, $S_{2}, K_{1}$ and $O_{1}$ are imposed in the second case. Usually, the
Table 1 Condition of incident waves (Tokyo Bay)

\begin{tabular}{|c|c|c|c|}
\hline Constituents & Amplitude (m) & Period(hour) & Phase delay(rad) \\
\hline$M_{2}$ & $a_{1}^{l}=0.21$ & $T_{1}=12.42$ & $\kappa_{1}=-5.8398$ \\
\hline$S_{2}$ & $a_{2}^{I}=0.15$ & $T_{2}=12.00$ & $\kappa_{2}=-5.6018$ \\
\hline$K_{1}$ & $a_{j}^{I}=0.14$ & $T_{3}=23.93$ & $\kappa_{3}=-1.3290$ \\
\hline$O_{1}$ & $a_{l}^{I}=0.10$ & $T_{4}=25.82$ & $\kappa_{4}=-1.3211$ \\
\hline
\end{tabular}

( YOKOHAMA)
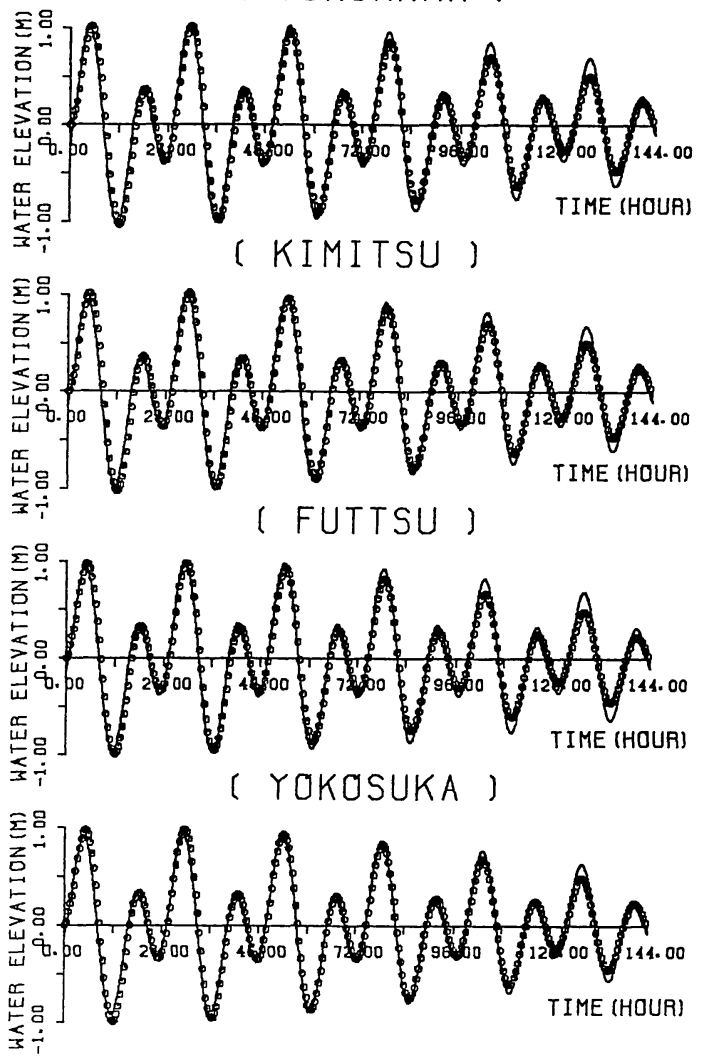

(०) : Present method, (-) : Observed data

Fig.12 Time variation of tidal level of main four constituents at the observations points

period, wave-direction and phase lag are known, but the amplitude of incident wave is unknown and have to be estimated. The identification method proposed by Kodama et al ${ }^{21)}$ is used for this purpose. The identification of the amplitude of incident waves is carried out based on the minimization of residuals between the computed water elevations and observed data which are recorded at fixed points in the flow field. For the computation, the time increment $\Delta t=15 \mathrm{~s}$. is employed.

a) The case of $M_{2}$ constituent of the tide

In this case the $M_{2}$ constituent of the tide is imposed as follows :

$$
\bar{\eta}^{I}=a_{I} \sin \frac{2 \pi}{T} t
$$




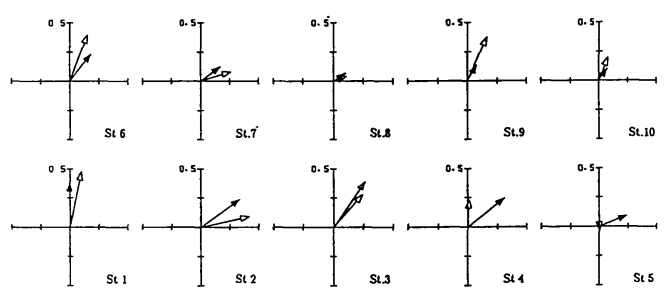

(Upper layer)
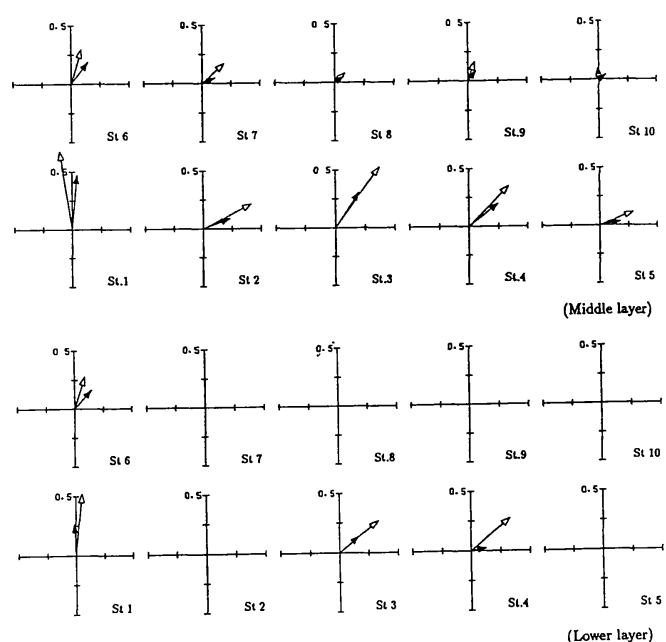

Maximum NW-going stream

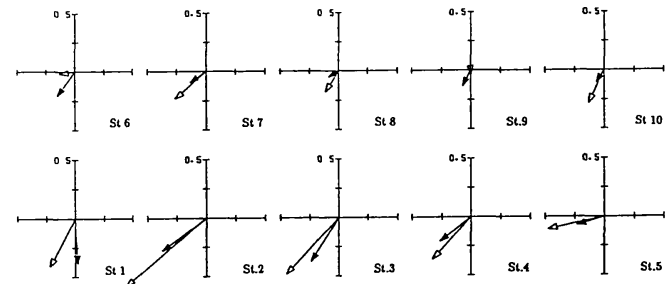

(Upper layer)
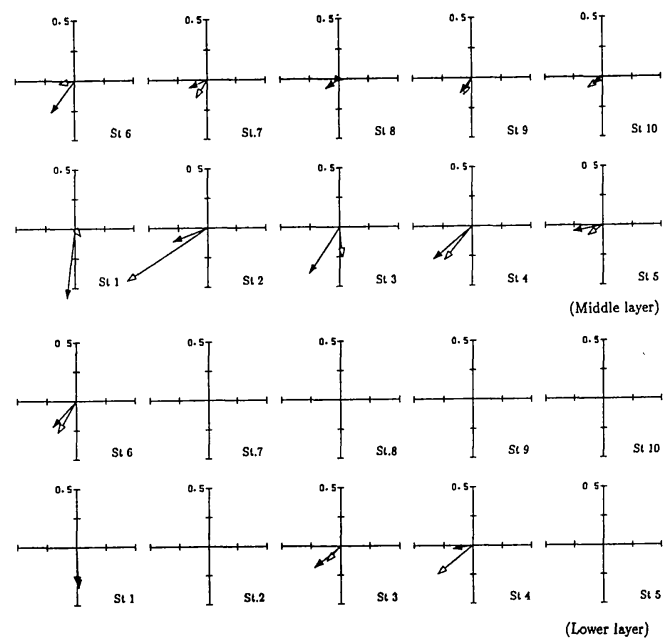

Maximum SE-going stream

—: Present method, ـ : Observed data, unit : knot

Fig.13 Comparison between the observed and computed velocity

in which $a_{I}=0.25 \mathrm{~m}$ was estimated by the identification method $^{21)}$ and a period, $T=12.42$ hours was assumed. The tide levels for the first 144 hours obtained by the present method and the observed tidal level are shown in Fig.10. The observed tidal level were generated referring to the harmonic constant table ${ }^{22)}$. The comparison shows that agreement between the two results is good in phase level. However, difference exist in amplitude for a particular case at Anegasaki.

The nature of obtained tidal current velocity for different stations for surface, middle and lower level has been explained in ellipses graph in Fig.11. These results agree well with the observed data ${ }^{23)}$ which have been measured in the period, August 25 to October 25, 1983, except the station 1 and 2 . At station 1, the direction of the present tidal current velocity does not match with the observed data, however its value shows good agreement, whereas at station 2 inverse behavior has been noted. It may be happened because of those locations and approximation of water depth by the comparatively rough mesh division. The actual fact is not clear in this stage.

b) The case of main four constituents of the tide

In this case, the main four constituents of the tide are imposed :

$$
\bar{\eta}^{I} \sum_{m=1}^{4} a_{m} \sin \left(\frac{2 \pi}{T_{m}} t-\kappa_{m}\right)
$$

where the constants are shown in Table 1. Amplitudes of the constituents were also estimated by the identification $\operatorname{method}^{21)}$. The time variations of tide level by the present method are compared in Fig.12 with the observed tidal levels ${ }^{22)}$. Both show good agreement. The computed velocities and the measured data $^{23)}$ which have been observed in a period, August 25 to October 25, 1983 are compared in Fig. 13. In both cases, Maximum North West stream and South East stream, the velocity by the present method are in good agreement with the measurements for surface, middle and lower levels. The computed flow pattern of both cases are illustrated in Fig.14. In the tidal current analysis of Tokyo Bay using the open boundary condition, the reasonable results can be obtained. However, it should be noted that this boundary condition is based on the assumption, the reflective wave propagates with the normal direction to the boundary. If this assumption is not realized, the wave direction of reflective component has to be estimated by some way.

\section{CONCLUDING REMARKS}

The finite element method to analyze a tidal current flow with the open boundary condition is 

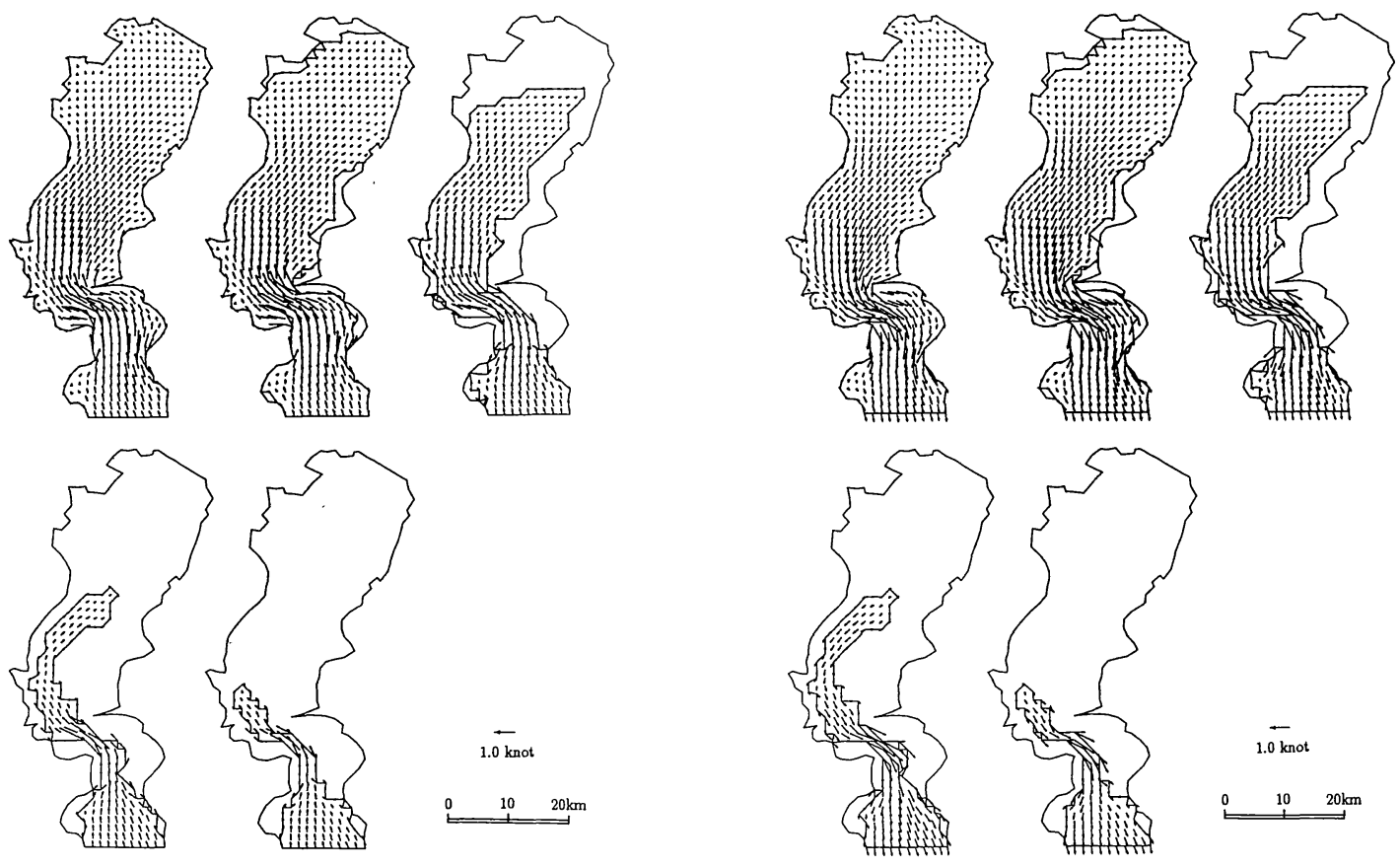

Maximum NW-going stream

Fig.14 Computed velocity distribution

presented. The main conclusions obtained are as follows :

1. The open boundary condition wich does not cause the spurious reflective wave on the openboundary is proposed for the multiple level flow model. This method is quite suitable for the practical analysis because it is adaptable for the arbitrary configuration of the open boundary and of shape of elements of which nodal points are located on the open boundary. Since the tidal wave is divided into the incident and reflective wave components, the method can easily handle the computations to investigate the effect of the modifications of the boundary shape.

2. The compartive study is carried out for a one dimensional channel model to show the applicability of this method. The results by the present method are in good agreement with the analytical solutions for linear cases.

3. The analysis of the tidal currents for Tokyo Bay is performed to verify the validity of the present method for a practical problem. The water elevations and velocities of the tide are computed, and compared with the observed data. The correspondence between the computed and observed results is well.

It is concluded that the present method is a useful and effective tool for the analyses of the periodical flow problems such as tidal currents.

\section{REFERENCES}

1) Holzs, K. P. : Numerical experiences from the computation of tidal waves by the finite element method, Eds., Pinder, et al. Finite Elements in Water Resources, Pentech Press, Plymouth, pp.419 431, 1976.

2) Kawahara, M., Kobayashi, M. and Nakata, K. : Multiple level finite element analysis and its applications to tidal current flow in Tokyo Bay, Appl. Math. Modelling, Vol.7, pp.197 211, 1983.

3) Murakami, K. : Numerical simulations on tidal currents by means of finite element method, Technical Note of Port \& Harbour Research Institute (Ministry of Transport, Japan), No.404, 1981 (in Japanese).

4) Leendertse, J. J., Alexander, R. C. and Liu, S. K. : A three dimensional model for estuaries and coastal seas : Vol. I, Principles of Computation, The Rand Corporation, R1417-OWRR, 1973.

5) Leendertse , J.J. and Liu, S. K. : A three dimensional model for estuaries and coastal seas : Vol. II , Aspects of Computation, The Rand Corporation, R-1764-OWRT, 1973.

6) Liu, S. K. and Leendertse , J. J.: A three dimensional model for estuaries and coastal seas : Vol. VI, Bristol Bay Simulations, The Rand Corporation, R-2405-NOAA, 1979.

7) Heaps, N. S. : Three-dimensional model for tides and surges with vertical eddy viscosity prescribed in two layersI . Mathematical formulation, Geophys. J. R. astr. Soc., Vol.64, pp.291 302, 1981. 
8) Heaps, N. S. and Jones, J. E. : Three-dimensional model for tides and surges with vertical eddy viscosity prescribed in two layers- II. Irish Sea with bed friction layer, Geophys. J. R. astr. Soc., Vol.64, pp.303 320, 1981.

9) Noye, B. J., May R. L. and Teubner, M. D. : Threedimensional numerical model of tides in spencer gulf, Ocean Management, Vol.6, pp.137 148, 1981

10) Bills, P. J. and Noye, B. J. : Verification of a threedimensional tidal model for coastal seas, Proc. of CTAC83, pp.394 410, 1983.

11) Wang, H. P. : Multi-level finite element hydrodynamical model of Block Island Sound, Finite Element in Water Resources (eds. G. Pinder et al.), Princeton University, Pentech Press, pp.4-69 4-93, 1975.

12) Kobayashi, M., Nakata,-K. and Kawahara, M. : A threedimensional multi-leveled finite element method for density current analysis, Finite Element Methods in Flow Problem, Vol. II (eds. R. H. Gallagher et al.), University of Calgary, pp.80 92, 1980.

13) Qian, Z., Nagata, Y. and Imm, S. : The boundary conditions at open boundaries for tidal calculations, Research Notes of Nearshore, Vol.21, pp.55 61, 1983 (in Japanese).

14) Smith, W. D. : A nonreflecting plane boundary for wave propagation problems, J. Comp. Phys. Vol.15, pp.492 503, 1974
15) Oliger, J. and Sundstrom, A. : Theoretical and practical aspects of some initial boundary value problems in fluid dynamics, SIAM, Vol.35, pp.419 446, 1978.

16) Engquist, B. and Majda, A. : Absorbing conditions for the numerical simulation of waves, Math. Comp. Vol.31, pp.629 651, 1977.

17) Bode, L. and Sobey, R. J. : Initial transients in long wave computations, J. Hydraulic Engineering, ȦSCE, Vol.111, pp.237 255, 1984.

18) Blumberg, A. and Kantha, L. : Open boundary condition for circulation models, J. Hydraulic Engineering, ASCE, Vol.110, pp.1371 1397, 1985.

19) Kawaharwa, M., Hirano, H., Tsubota, K. and Inagaki, K. : Selective lumping finite element method for shallow water flow, Int. J. Numer. Meth. Fluids, Vol.12, pp.331 351, 1982.

20) Maritime Safety Agency : Maritime Chart of Tokyo Datum, No.90, 1984.

21) Kodama, T., Kawasaki, T. and Kawahara, M. : A finite element method for shallow water equation including open boundary condition, Int, J. Numer. Meth. Fluids, Vol.13, pp.939 953, 1991.

22) Maritime Safety Agency : Harmonic constant table, 1989.

23) Port \& Harbour Research Institute : Report of investigation of tidal current in Tokyo Bay, 1983.

(Received November 20, 1991)

\section{無反射性開境界条件を用いた多層有限要素法による潮流解析 児玉敏雄・川原睦人}

本論文では, 開境界条件を考慮した多層モデルによる潮流解析の数値計算手法を提案 する. 開境界に対して疑似の反射波の生じない入射境界条件の処理方法を示した. 開境 界において領域外に透過すべき反射波成分を『部分領域』を導入することにより数值的 に求めるという点が本手法の特徵である. 本手法の適用性を解析解との比較および東京 湾の潮流解析を行うことにより示した. 